\title{
EFECTO DE ONDAS SONICAS EN LA ACCIÓN DE TERMITAS SUBTERRÁNEAS SOBRE PIEZAS DE MADERA DE DIFERENTES ESCUADRIAS
}

Alejandro Bozo González y José Tomas Karsulovic C. ${ }^{2}$

\section{RESUMEN}

La introducción accidental de la termita subterránea (Reticulitermes $s p$.) al territorio nacional, hace más de 15 años, esta ocasionando un problema que comienza lentamente a vislumbrarse. Estos insectos se alimentan fundamentalmente de celulosa y pueden llegar a ocasionar daños significativos en las construcciones. Esto puede ser una amenaza a las pretensiones de convertir a la madera, generada en el pais, en la solución para el problema habitacional que se tiene hoy en dia a nivel nacional. Los efectos de las termitas subterráneas se manifiestan diariamente en las comunas de la Región Metropolitana y desde algún tiempo han empezado a manifestarse en comunas de regiones vecinas (Valparaiso y O'Higgins).

El Departamento de Ingenieria de la Madera de la Universidad de Chile, ha desarrollado una linea de investigaciones tendiente a detectar y controlar la termita subterránea, utilizando técnicas no destructivas como son la aplicación de ondas sónicas y ultrasónicas. Los resultados presentados en este articulo se enmarcan en esta linea de investigación.

El objetivo de este estudio es determinar el efecto de ondas sónicas en el comportamiento de termitas subterráneas durante el proceso de infestación en el interior de piezas de madera de pino radiata (Pinus radiata D.Don) de diferentes escuadrias.

La metodologia consistió básicamente en exponer a la acción de las termitas madera de pino radiata en escuadrias de $40 \times 45 \mathrm{~mm}, 40 \times 70 \mathrm{~mm}$ y $40 \times 90 \mathrm{~mm}$, en un largo de $200 \mathrm{~mm}$ y a un contenido de humedad de $12 \%$. Estas piezas fueron colocadas en contenedores de vidrio de $12 \times 7 \mathrm{~cm}$ de base y $25 \mathrm{~cm}$ de altura, conformando cada una de ellas un mini termitero con 250 obreras y 15 individuos entre soldados y ninfas. Para el manejo del material biológico se siguió las prescripciones de la norma española UNE 56-410-92. Basándose en los resultados obtenidos en estudios previos se procedió a aplicar ondas sónicas en la parte superior de las piezas de madera. Cada ensayo se efectuó con tres repeticiones y un testigo. Los resultados registrados fueron la tasa de alimentación y la supervivencia de las termitas.

Los resultados revelan que existe una disminución de la tasa de alimentación con la aplicación de ondas sónicas para todas las escuadrias estudiadas.

Palabras claves: Termitas subterráneas, ondas sónicas, pino radiata.

1 Departamento de Ingenieria de la Madera, Facultad de Ciencias Forestales, Universidad de Chile. CHILE. abozo@uchile.cl

2 Departamento de Ingenieria de la Madera, Facultad de Ciencias Forestales, Universidad de Chile. CHILE. Ikarsulo@uchile.cl 


\section{EFFECT OF SONIC WAVES IN THE ACTION OF SUBTERRANEAN TERMITES ON PIECES OF WOOD OF DIFFERENT SIZES}

\section{SUMMARY}

The accidental introduction of the subterranean termite (Reticulitermes sp.) to the national territory, since 15 years, this causing a problem that slowly begins to glimpse. These insects feed fundamentally on cellulose and can get to cause significant damages in the constructions. This can be a threat to the pretensions to turn to the wood, generated in the country, in the solution for the housing problem that Chile has nowadays at national level. The effects of the subterranean termites are pronounced daily in the communes of the Metropolitan Region and from some time they have begun to pronounce in communes of neighboring regions (Valparaiso and O'Higgins).

The Department of Wood Engineering of the University of Chile has developed a line of nondestructive technical investigations to detect and control the subterranean termite using the application of sonic and ultrasonic waves. The results presented in this paper are framed in this line of investigation.

The Objective of this study is to determine the effect of sonic waves in the behavior of subterranean termites during the process of infestation inside wood pieces of Radiata Pine (Pinus radiata D.Don) of different size.

The methodology consisted basically of exposing to the action of the termites wood of Radiata Pine of following cross section: $40 \times 45 \mathrm{~mm} ; 40 \times 70 \mathrm{~mm}$ and $40 \times 90 \mathrm{~mm}$ in a length of $200 \mathrm{~mm}$ to a moisture content of $12 \%$. These pieces were placed in glass containers of 12 $\times 7 \mathrm{~cm}$ of base and $25 \mathrm{~cm}$ of height, conforming each one of them a mini termite nest with 250 workers and 15 individuals between soldiers and nymphs. For the handling of the bacteriological agents the prescriptions of the Spanish norm were followed UNE 56-410-92. Being based on the results obtained in previous studies it was come to apply sonic waves in the superior part of the wood pieces. Each test took place with three repetitions and a control. The registered results were the rate of feeding and the survival of the termites.

The results revealed that exist a diminution of the rate of feeding with the application of sonic waves for all wood sizes studied.

Key words: Subterranean termites, sonic waves, Radiata Pine 


\section{INTRODUCCIÓN}

Los resultados que se reportan aqui corresponden a una parte de una investigación que tiene por finalidad la aplicación de ondas sónicas para crear una barrera fisica a la acción de las termitas subterráneas en construcciones en madera. Si bien las termitas juegan un importante rol ecológico en la naturaleza, siendo la celulosa su principal fuente de alimentación, éstas constituyen un grave agente de deterioro para la madera en servicio.

Hasta hace algunos años en Chile existian solo dos familias de termitas, Kalotermidae y Termopsidae agregándose, con la introducción de la termita subterránea, la familia Rhinotermitidae a la cual pertenece la especie Reticulitermes flavipes Kollar, identificada inicialmente en Chile como Reticulitermes hesperus Bank, la cual seria originaria de Estados Unidos e introducida en Chile en la década de los setenta. Es reconocido el hecho que las especies del género Reticulitermes son la mayor plaga que se encuentra infestando estructuras en madera en numerosos paises del mundo, provocando cuantiosas pérdidas.

Se ha detectado en la zona central de Chile una vasta infestación, con un significativo incremento durante los últimos cinco años, corroborado por las numerosas denuncias de la presencia de termitas en viviendas, las cuales en su gran mayoria corresponden a construcciones destinadas a la habitación de personas de niveles sociales de menores recursos económicos. que son construidas integramente de madera las que son utilizadas sin ningún tipo de tratamiento de preservación y que no siguen además ninguna normativa de construcción.

La erradicación total de las termitas no es posible y los esfuerzos deben orientarse a controlar la existencia de la población de insectos y limitar o disminuir los riesgos a las construcciones, a través de la implementación de estrategias integradas de manejo de la plaga (Morris, 2000). Para un adecuado manejo de las termitas es necesario disponer de métodos de prevención y control. Para la prevención se utilizan diferentes técnicas, consistente en el establecimiento de barreras fisicas y quimicas. En general, el uso de barreras fisicas está concebido para tratamientos de preconstrucción de una edificación (uso de arenas, mallas de acero, plásticos impregnados, etc.). Las barreras quimicas se utilizan tanto como tratamientos preventivos como curativos en pre y post construcción y tienen por objetivo la exclusión de las termitas subterráneas de las estructuras.

Los métodos curativos consisten en la aplicación de tratamientos quimicos y no quimicos, como por ejemplo, la utilización de dióxido de carbono (Delate et. al, 1995), aire caliente (Woodrow y Grace, 1998), nitrógeno liquido (Lewis 1997). Los tratamientos químicos en áreas localizadas consisten en la perforación y posterior inyección de insecticida en las galerias construidas por las termitas.

Para la aplicación de métodos curativos es necesario disponer de sistemas de inspección o detección de la actividad de las termitas en elementos en servicio. Para éste objetivo se ha desarrollado diferentes técnicas, tales como emisión acústica (Scheffranhm, 1993; Lemaster, 1997; Yanase, 1998; Mankin, 2002), ondas de esfuerzo (Ross et. al. 1997; De Groot et. al. 1998), ultrasonido (Wilcox, 1998), detección de gases que emiten las termitas durante su metabolismo (metano y dióxido de carbono), imágenes infrarrojas, microondas, rayos $\mathrm{x}$, etc. 
Este trabajo tiene la finalidad de efectuar un nuevo aporte a las técnicas ya existentes para el control de termitas. Se postula que las termitas pueden ser susceptibles a la acción de ondas mecánicas, a una determinada frecuencia e intensidad de irradiación, pudiendo ello producir alteraciones en sus mecanismos de comunicación, alimentación u otros cambios biofísicos y/o bioquimicos que ocasionen efectos inmediatos o mediatos en su comportamiento o sobrevivencia.

\section{OBJETIVOS}

El objetivo general determinar el efecto de ondas acústicas sobre el comportamiento de termitas subterráneas durante el ataque de estas en madera de pino radiata de diferentes escuadrias.

Los objetivos especificos son determinar el efecto en la tasa de alimentación de las termitas subterráneas cuando están sometidas al efecto de ondas sónicas y evaluar la mortalidad de las termitas subterráneas sometidas al efecto de ondas sónicas durante un periodo de dos meses.

\section{MATERIAL Y MÉTODO}

\section{Recolección y Manutención de Termitas}

Las termitas utilizadas en los ensayos provinieron de una colonia ubicada en la parte central de la ciudad de Santiago. La captura se efectuó mediante cebos diseñados y construidos para éste efecto, utilizando tubos de PVC de $75 \mathrm{~mm}$ de diámetro y $250 \mathrm{~mm}$ de largo, rellenos con cartón corrugado como fuente de alimentación y tapados en ambos extremos. A los tubos se les practicaron ranuras de $5 \mathrm{~mm}$ de ancho a través de su periferia en dirección transversal para permitir la entrada de las termitas (Figura $\mathrm{N}^{\circ} 1$ ). Los cebos fueron enterrados en los lugares infestados durante un periodo de 20 dias. 


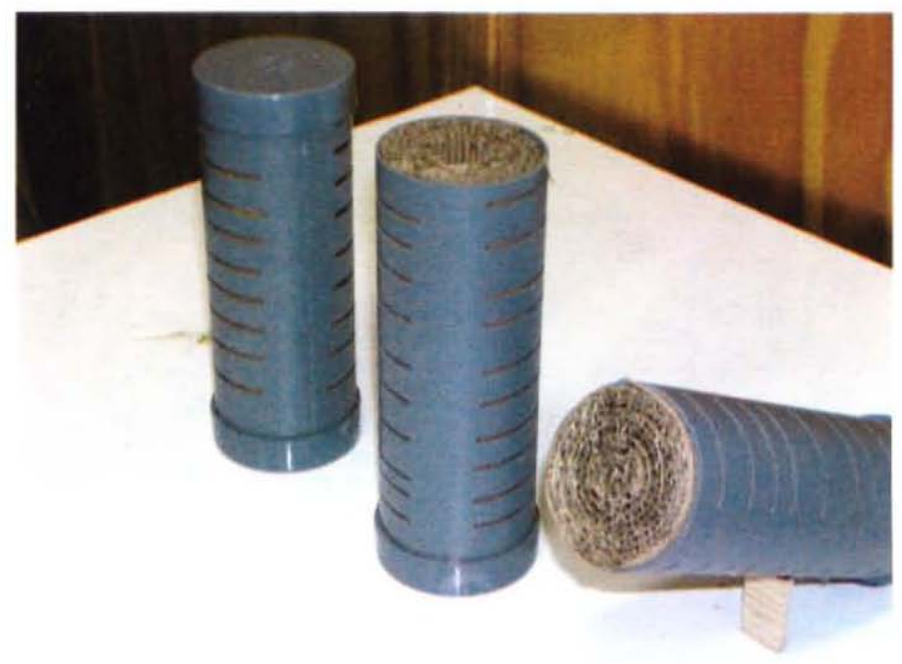

Figura $\mathrm{N}^{\circ} 1$

CEBOS DE CAPTURA

Con las termitas capturadas, obreras y soldados, fueron establecidas cuatro colonias, las que fueron instaladas en una sala climatizada con ambiente controlado de temperatura, $24^{\circ} \mathrm{C}$, y humedad relativa de $70 \%$ a $80 \%$, de modo de otorgarle a los insectos las condiciones óptimas para su manutención y desarrollo.

La constitución de los termiteros se realizó mediante receptáculos de vidrio de $60 \mathrm{~cm}$ de longitud por $45 \mathrm{~cm}$ de ancho y $30 \mathrm{~cm}$ de alto, con tapa y orificio de aireación cubierto con rejilla metálica (Figura $\mathrm{N}^{\circ}$ 2). En su interior se introdujo un sustrato formado de gravilla mezclada con tierra, arena de cuarzo y material inductor consistente de pequeños trozos de madera de pino radiata. Para mantener la humedad del sustrato se adicionó diariamente una cantidad de agua destilada. Como fuente de alimentación se instaló dentro de los termiteros paquetes de 10 tablillas de madera de pino radiata de $5 \mathrm{~mm}$ de espesor, $60 \mathrm{~mm}$ de ancho y $200 \mathrm{~mm}$ de largo, unidas entre si con amarras de alambre de acero. De acuerdo a los requerimientos de los ensayos se fue extrayendo los paquetes y coleccionando las termitas en las cantidades necesarias, para luego retornar los paquetes al termitero. 


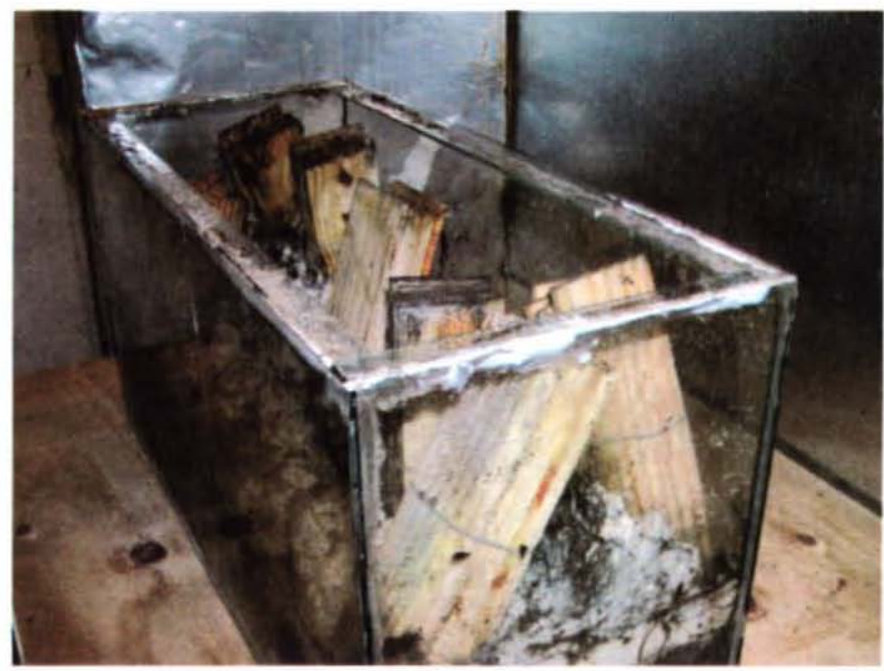

Figura $\mathrm{N}^{\circ} 2$

TERMITERO UTILIZADO EN LABORATORIO

\section{Equipos y Accesorio}

Generador de frecuencias (Aagilent 33220A).

Amplificador (Peavy cs $1200 \mathrm{X}$ ).

Altavoces.

Cámara climatizada.

Balanza.

\section{Método}

El método consistió básicamente en exponer a la acción de las termitas madera de pino radiata de las siguientes escuadrias: $40 \times 45 \mathrm{~mm}, 40 \times 70 \mathrm{~mm}$ y $40 \times 90 \mathrm{~mm}$, en un largo de $200 \mathrm{~mm}$ (Figura $\mathrm{N}^{\circ}$ ) a un contenido de humedad de $12 \%$. 


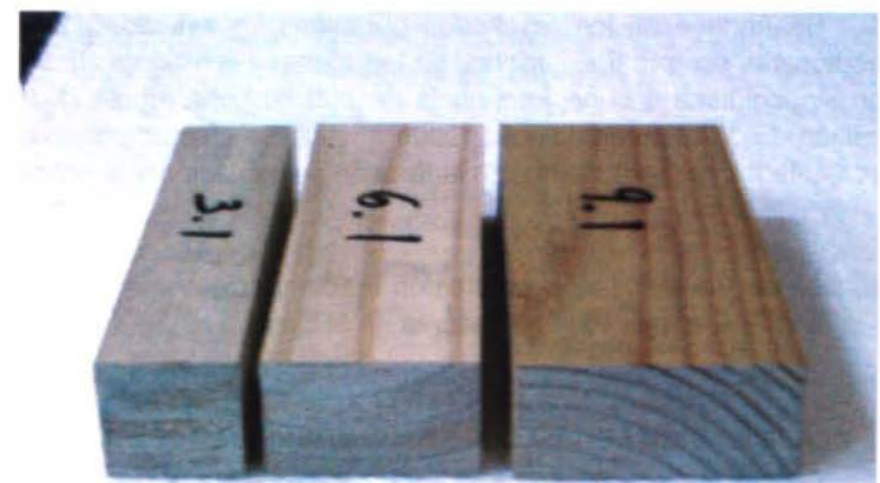

Figura $\mathrm{N}^{\circ} 3$

PIEZAS DE MADERA DE DIFERENTES ESCUADRIAS

Estas piezas fueron colocadas en contenedores de vidrio de $12 \times 7 \mathrm{~cm}$ de base y $25 \mathrm{~cm}$ de altura (Figura $\mathrm{N}^{\circ} 4$ ), conformando cada una de ellas un mini termitero con 250 obreras y 15 individuos entre soldados y ninfas.

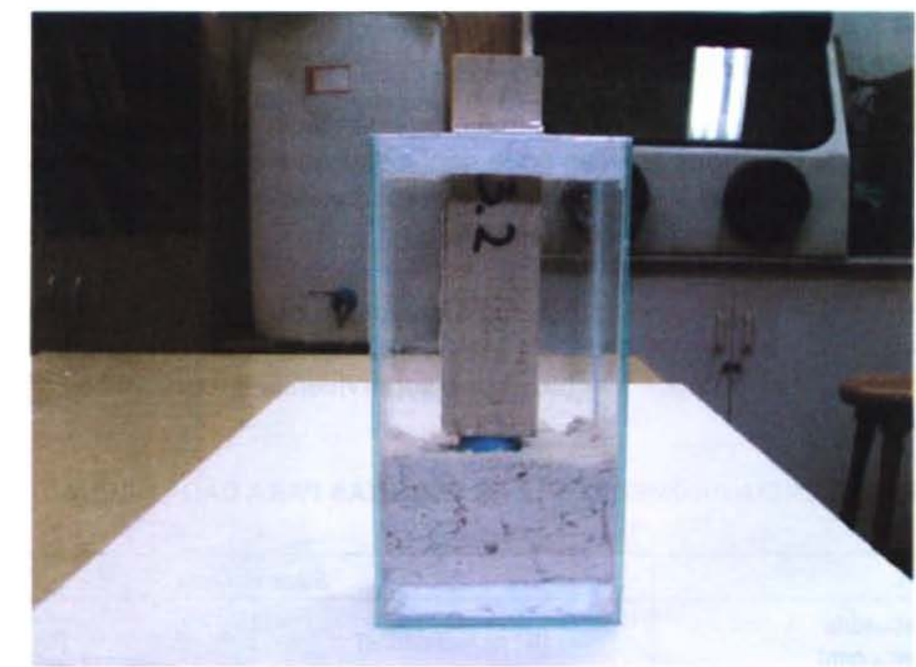

Figura $\mathrm{N}^{\circ} 4$

MINI TERMITERO DISEÑADO PARA LA REALIZACIÓN DE LOS ENSAYOS

Para el manejo del material biológico se siguió las prescripciones de la norma española 
UNE 56-410-92. Basándose en los resultados obtenidos en estudios previos se procedió a aplicar ondas sónicas en la parte superior de las piezas de madera. Las ondas sónicas aplicadas fueron sinusoidales de una frecuencia de $200 \mathrm{~Hz}$, una amplitud de $1.5 \mathrm{Vpp}$ y un tiempo de aplicación de 30 minutos diarios. Cada ensayo se efectuó con tres repeticiones y un testigo. Los resultados registrados fueron la tasa de alimentación y la sobrevivencia de las termitas.

La evaluación de los resultados se efectuó mediante un análisis cualitativo, según lo establecido por la Norma Europea EN 118 descrita en el Cuadro $\mathrm{N}^{\circ} 1$.

\section{Cuadro $\mathrm{N}^{\circ} 1$}

\section{CLASIFICACIÓN DEL ATAQUE SEGÚN INTENSIDAD DEL DAÑO}

\begin{tabular}{|c|c|}
\hline Grado de Ataque & Descripción \\
\hline 0 & Ningùn ataque \\
\hline 1 & Tentativa de ataque: Arañazos o roeduras superficiales cuya profundidad no se puede medir. \\
\hline 2 & $\begin{array}{l}\text { Alaque ligero: Ataque superficial (menos de } 1 \mathrm{~mm} \text { ) y limitado en extensión a } 1 / 4 \text { de la superficie expuesta } \\
\text { como máximo. o una perforación única de profundidad infenor a } 3 \mathrm{~mm} \text {. sin que exista otra tasa de ataque. }\end{array}$ \\
\hline 3 & $\begin{array}{l}\text { Ataque medio Ataque superficial (menos de } 1 \mathrm{~mm} \text { ) que se extiende a mas de } 1 / 4 \text { de la superficie } \\
\text { expuesta o erosion (de } 1 \text { a } 3 \mathrm{~mm} \text { ) sobre una superficie inferior o igual a } 1 / 4 \text { de la expuesta o perforaciones } \\
\text { puntuales superiores a } 3 \mathrm{~mm} \text {, pero que no se extiendan en cavernas o no atraviesen. }\end{array}$ \\
\hline 4 & $\begin{array}{l}\text { Ataque fuerte: Erosión sobre más de } 1 / 4 \text { de la superficie expuesta o ataque penetrante supenor a } 3 \mathrm{~mm} \\
\text { extendiendose en cavernas en el interior de la probeta o sin extenderse en cavernas. pero atravesàndola. }\end{array}$ \\
\hline
\end{tabular}

Fuente: Norma Europea EN 118, 1992

\section{RESULTADOS Y DISCUSIÓN}

Los resultados de estos ensayos permitieron cuantificar el porcentaje de supervivencia y evaluar el efecto de las ondas sónicas en la capacidad de alimentación de las termitas. En el Cuadro $\mathrm{N}^{\circ} 2$ se presentan los porcentajes de sobrevivencia promedio para las distintas escuadrias utilizadas y para las probetas testigos.

La Norma EN 118 fija como válidos los ensayos en que dos de tres testigos alcanzan una supervivencia mayor a un $50 \%$ de los individuos. Lo que en este ensayo fue logrado, puesto que en las termitas testigos se obtuvo una supervivencia promedio de $64 \%$.

\section{Cuadro $\mathrm{N}^{\circ} 2$}

\section{SUPERVIVENCIA PROMEDIO DE LAS TERMITAS PARA CADA ESCUADRIA}

\begin{tabular}{|c|c|c|}
\hline & \multicolumn{2}{|c|}{ Supervivencia } \\
\hline $\begin{array}{c}\text { Escuadria } \\
{[\mathbf{m m} \times \mathbf{~ m m}]}\end{array}$ & (N $^{\circ}$ de Individuos) & (\%) \\
\hline $40 \times 45$ & 137 & 54,8 \\
\hline $40 \times 70$ & 132 & 52,8 \\
\hline $40 \times 90$ & 139 & 55.6 \\
\hline Testigos & 160 & 64,0 \\
\hline
\end{tabular}


Para las tres escuadrias la supervivencia fue muy similar y siempre superior al $50 \%$, el ataque a la madera correspondió a un ataque fuerte como se puede apreciar en el Cuadro $\mathrm{N}^{\circ}$ 3 donde se presenta los grados de ataque promedio sufrido por las probetas según Norma EN 118.

\section{Cuadro $\mathrm{N}^{\circ} 3$}

GRADO DE ATAQUE PROMEDIO SUFRIDO POR LAS PROBETAS SEGÚN NORMA EN 118

\begin{tabular}{|c|c|}
\hline $\begin{array}{c}\text { Escuadria } \\
{[\mathbf{m m} \times \mathbf{~ m m}]}\end{array}$ & Grado de ataque \\
\hline $40 \times 45$ & 4 \\
\hline $40 \times 70$ & 4 \\
\hline $40 \times 90$ & 4 \\
\hline Testigos & 4 \\
\hline
\end{tabular}

La supervivencia de las diferentes escuadrias fue menor al testigo en casi 10 puntos porcentuales por lo cual es posible deducir decir que esto se debe a la aplicación de las ondas sónicas. Sin embargo, la reducción en la supervivencia no es tan significativa y puede deberse a la interacción de diferentes factores.

Testimonios gráficos del ataque en las diferentes escuadrias pueden ser observados en las imágenes mostradas en la Figura $\mathrm{N}^{\circ} 5$.

En el Cuadro $\mathrm{N}^{\circ} 4$ se presenta los resultados del grado de alimentación de las termitas después de irradiación, expresados como consumo de alimento, asociados a cada escuadria. Los valores corresponden a cifras promedio para cada uno de los tratamientos, con tres repeticiones. 

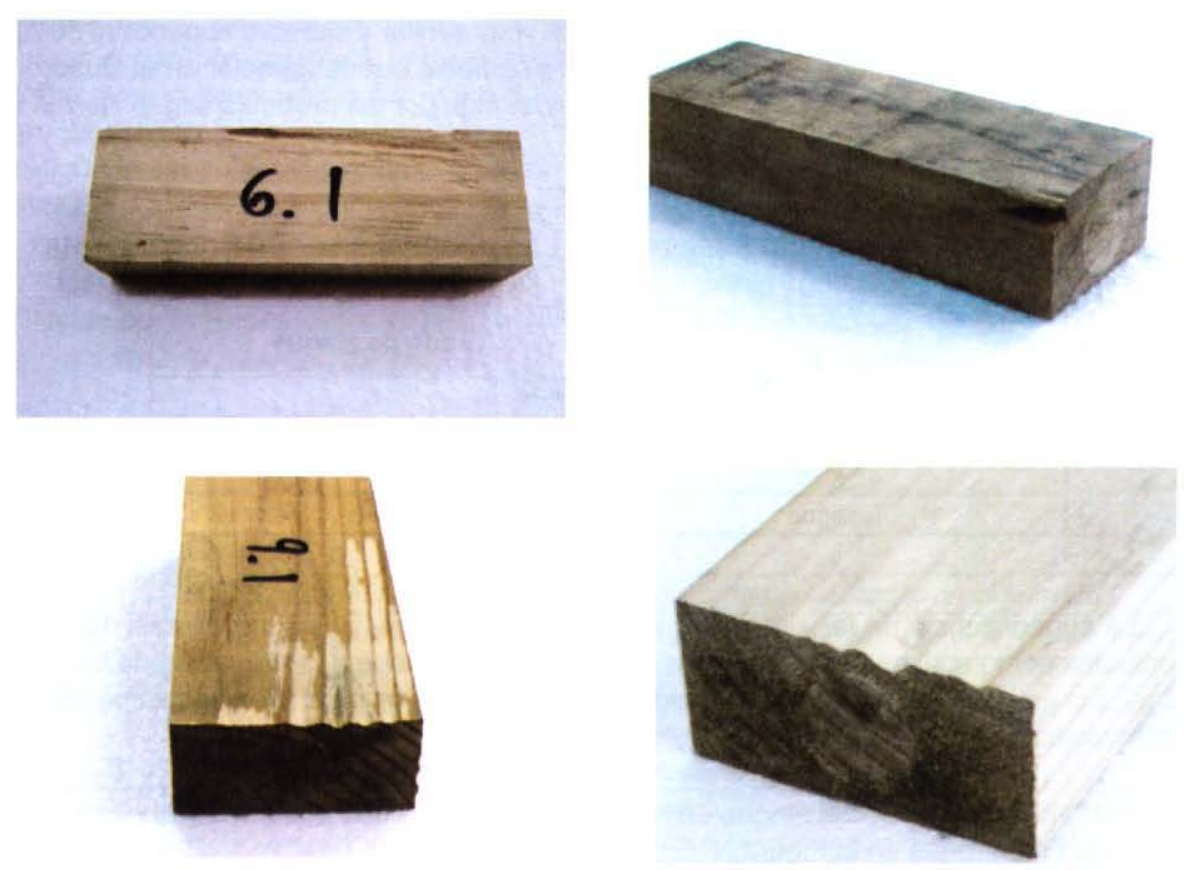

Figura $\mathrm{N}^{\circ} 5$

EFECTO DE LA ACCIÓN DE LAS TERMITAS EN LAS DIFERENTES ESCUADRIAS

Cuadro $\mathrm{N}^{\circ} 4$

CONSUMO DE ALIMENTO, SEGÚN ESCUADRIA

\begin{tabular}{|c|c|c|}
\hline $\begin{array}{c}\text { Escuadria } \\
(\mathbf{m m} \times \mathbf{~ m m})\end{array}$ & Estadigrafos & $\begin{array}{c}\text { Consumo de Alimento } \\
\mathbf{( g )}\end{array}$ \\
\hline \multirow{2}{*}{$40 \times 45$} & Media & 0,981 \\
& Des. Estándar & 0,166 \\
& COV \% & 16,92 \\
\hline \multirow{2}{*}{$40 \times 70$} & Media & 1,021 \\
& Des. Estándar & 0,090 \\
& COV \% & 8,81 \\
\hline \multirow{2}{*}{$40 \times 90$} & Media & 1,521 \\
& Des. Estándar & 0,109 \\
& COV \% & 7,17 \\
\hline
\end{tabular}

En el Cuadro $\mathrm{N}^{\circ} 5$ se presenta los resultados del consumo de alimento de los testigos, para cada una de las repeticiones. Cabe hacer notar que el COV de los testigos es claramente mayor (29, 3 \%) que los presentados en la tasa de alimentación de las distintas escuadrias (11 $\%$ promedio). 


\section{Cuadro $\mathrm{N}^{\circ} 5$ \\ CONSUMO DE ALIMENTO DE TERMITAS TESTIGO}

\begin{tabular}{|c|c|}
\hline Repetición & $\begin{array}{c}\text { Consumo de Alimento } \\
(\mathbf{g})\end{array}$ \\
\hline 1 & 1,168 \\
\hline 2 & 1,561 \\
\hline 3 & 2,110 \\
\hline Media & 1,613 \\
\hline Des. Estándar & 0,473 \\
\hline COV(\%) & 29,3 \\
\hline
\end{tabular}

\section{CONCLUSIONES}

Los ensayos sónicos mostraron claras evidencias que la irradiación para las tres escuadrias constituye una alteración en la tasa de alimentación de las termitas.

Los resultados revelaron que existe una disminución de la tasa de alimentación con la aplicación de ondas sónicas para todas las escuadrias estudiadas.

La tasa de supervivencia de las termitas sometidas a irradiación sónica es más baja que los testigos, debido muy probablemente al efecto de las ondas sónicas aplicadas.

El COV de la supervivencia fue claramente más alto para los testigos que para las termitas sometidas a irradiación sónica.

A la luz de los resultados obtenidos se concluye que es necesario realizar un mayor número de ensayos utilizando otras frecuencias y amplitudes.

\section{AGRADECIMIENTOS}

Los autores agradecen a FONDECYT por el financiamiento del Proyecto $\mathrm{N}^{\circ} 1040726$, en cuyo contexto se realizó éste estudio.

\section{REFERENCIAS}

De Groot, RC., Ross, RJ. \& Nelson, WT., 1998. Non-destructive assessment of wood decay and termite attack in southerm pine sapwood. Wood Protection 3: 25-34.

Delate, KM., Grace, FK. \& Armstrong, JW., 1995. Carbon dioxide as a potential fumigant for termite control. Pesticide Science 44: 357-361.

Lemaster, R.I., Beall, F.C. and Lewis, V.R., 1997. Detection of termites with acoustic emission. For. Prod. J.47:75 -79 
Lewis, VR., 1997. Alternative control strategies for termites. Journal of Agricultural Entomology 14: 291-307.

Mankin, RW, Osbrink, WL., O FM \& Anderson, JB., 2002. Acoustic detection of termite infestations in urban trees. Journal Economique Entomology 95: 981-988.

Morris, PI., 2000. Integrated control of subterranean termites: The 65 approach group leader-Durability and protection group. Forintek, Canada Corporation, Vancouver.

Ross, RJ., De Groot, RC., Nelson, WJ. \& Le Bow, PK., 1997. The relationship between stress wave transmission characteristics and the compressive strength of biologically degraded woods. Forest Products Journal 47: 89-93.

Scheffrahn, RH., Robbins, WP., Busey, P., Su, NY. \& Mueller, RK., 1993. Evaluation of a novel handherd, acoustic emissions detector to monitor termites (/soptera: Rkalotermitidae, Rhinotermitidae) in wood. Journal of Economic Entomology 86: 1720-1729.

Wilcox, W. and Wayne., 1988. Detection of early stages of wood decay with ultrasonic pulse velocity. Forest Prod. I., 38(5): 68-73

Woodrow, RJ. \& Grace, JK., 1998. Thermal tolerances of four termites species (Isoptera: Rhinotermitidae, Kalotermitidae). Sociobiology 32: 17-25.

Yanase, Y., Fuji, Y., Okumura,Y. Imamura, T. \& Yoshimura, T., 1998. Detection of AE generated by the feeding activity of termites using PDVF. Forest Product Jornal 48: 43-46. 